\title{
A SET OF CRITERIA FOR THE SUMMABILITY OF DIVERGENT SERIES.
}

BY PROFESSOR WALTER B. FORD.

(Read before the Chicago Section of the American Mathematical Society, April 9, 1909.)

1. If the series

\section{Introduction.}

$$
u_{0}+u_{1}+u_{2}+\cdots+u_{n}+\cdots
$$

be divergent but of indeterminacy $r$ in Cesàro's sense, its sum is defined by the relation

(2) $s=\lim _{n=\infty} \frac{f(n, r) s_{0}+f(n-1, r) s_{1}+\cdots f(1, r) s_{n-1}+f(0, r) s_{n}}{f(n, r)+f(n-1, r)+\cdots f(1, r)+f(0, r)}$

where

$f(n, r)=r(r+1) \cdots(r+n-1) / n !(r \geqq 1) ; s_{n}=u_{0}+u_{1}+\cdots+u_{n}$ and where $r$ is taken as the smallest integer for which the indicated limit exists. Since it is a necessary condition for inde-terminacy $r$ that

$$
\lim _{n=\infty}\left(\frac{u_{n}}{n^{r}}\right)=0 \text { * }
$$

it follows that but few series (1) are summable by (2) for any value of $r$ however large, and hence that summability in Cesàro's sense is of relatively rare occurrence. It is therefore proposed in the present paper to show how an extended set of criteria, of which (2) affords a special type, may be constructed so as to provide an ascending scale of sharpness for the testing of summability. We shall show that such criteria may be constructed so as to bear a close analogy to the familiar logarithmic criteria for testing the convergence or divergence of a given series.

\section{Formulation of Problem and Theorem.}

2. In order to state the problem in more accurate terms we recall that the essential characteristic of the sum formula (2),

* Cf. Bromwich, Infinite Series, p. 318 (1908). 
or of any other general sum formula, is that it shall give $\lim _{n=\infty} s_{n}$ whenever the series (1) is convergent. Also we note that by increasing $r$ in (2) we increase the order of infinitude of its denominator when $n=\infty$ and thereby increase the range of applicability of the formula. The following problem, which is the one we shall undertake, is therefore suggested: To determine an infinite sequence of functions

$$
f_{0}(n, r), f_{1}(n, r), f_{2}(n, r), \cdots, f_{p}(n, r), \cdots
$$

each dependent upon the variable $n$ and the parameter $r$ and having the following characteristics:

$$
\lim _{n=\infty} \frac{\sum_{m=0}^{n} f_{p}(n-m, r) s_{m}}{\sum_{m=0}^{n} f_{p}(n-m, r)}=\lim _{n=\infty} s_{n}
$$

whenever the series (1) is convergent;

$$
f_{0}(n, r)=\frac{r(r+1) \cdots(r+n-1)}{n !} \quad \text { (case of Cesàro); }
$$

(c) $\lim _{n=\infty} \frac{\sum_{m=0}^{n} f_{p}(m, r)}{\sum_{m=0}^{n} f_{p}(m, r+1)}=0$,

(d) $\lim _{n=\infty} \frac{\sum_{m=0}^{n} f_{p}(m, r)}{\sum_{m=0}^{n} f_{p+1}(m, 1)}=0$.

Any function of the sequence (3) will thus furnish an infinite subset of criteria having an increasing range of applicability with increasing $r$ [see (c)], while in the case of a series (1) for which a given function of the sequence gives no meaning to the first member of $(a)$ for any value whatever of $r$, we may at once obtain more powerful criteria by increasing $p$ [see $(d)]$. Moreover, by virtue of $(b)$ the extended set of criteria thus obtained will form a generalization of Cesàro's original formula (2).

In this connection, we shall now establish the following

THEOREM. As a function $f_{p}(n, r)$ satisfying $(a),(b),(c),(d)$ and therefore furnishing a set of criteria of summability for divergent series we may take

$$
f_{p}(n, r)=\frac{r(r+1)}{r+\log _{p} n} \prod_{n=2}^{n} \frac{r+\log _{p} n}{\log _{p} n}
$$


where $\log _{0} n=n$ and $\log _{p} n=\log \log \ldots \log n$ ( $p$ times); $p=1,2,3, \cdots$.

3. Proof. We shall first establish the following lemma : Let $c_{0}, c_{1}, c_{2}, \cdots, c_{n}$ and $C_{0}, C_{1}, C_{2}, \cdots, C_{n}$ be two sequences of quantities of which the elements of the latter are real and positive. If $\lim _{n=\infty} c_{n}=g$ and

(4) $\quad \lim _{n=\infty} \frac{C_{n}+C_{n-1}+\cdots+C_{n-m}}{C_{n}+C_{n-1}+\cdots+C_{0}}=0, m=$ fixed integer $\geqq 0$, then

$$
\lim _{n=\infty} \frac{C_{n} c_{0}+C_{n-1} c_{1}+\cdots+C_{0} c_{n}}{C_{n}+C_{n-1}+\cdots+C_{0}}=g *^{*}
$$

In fact we shall then have $c_{n}=g+\epsilon_{n} ; \lim _{n=\infty} \epsilon_{n}=0$, so that we may write

where

$$
\frac{C_{n} c_{0}+C_{n-1} c_{1}+\cdots+C_{0} c_{n}}{C_{n}+C_{n-1}+\cdots+C_{0}}-g=X(n)+Y(n)
$$

$$
\begin{aligned}
& X(n)=\frac{C_{n} c_{0}+C_{n-1} c_{1}+\cdots+C_{n-m} c_{m}-g\left(C_{n}+C_{n-1}+\cdots+C_{n-m}\right)}{C_{n}+C_{n-1}+\cdots+C_{0}}, \\
& Y(n)=\frac{\epsilon_{m+1} C_{n-m-1}+\epsilon_{m+2} C_{n-m-2}+\cdots+\epsilon_{n} C_{0}}{C_{n}+C_{n-1}+\cdots+C_{0}} .
\end{aligned}
$$

But if $C$ represent the largest of the quantities $\left|c_{0}\right|,\left|c_{1}\right|$, $\cdots,\left|c_{m}\right|$, we shall have

$$
|X(n)| \leqq(C+g) \frac{C_{n}+C_{n-1}+\cdots+C_{n-m}}{C_{n}+C_{n-1}+\cdots+C_{0}}
$$

and hence $\lim _{n=\infty} X(n)=0$ by (4).

Again, corresponding to an arbitrarily small positive quantity $\epsilon$ we shall have

$$
\begin{aligned}
|Y(n)| \leqq & \epsilon \stackrel{C_{n-m-1}+C_{n-m-2}+\cdots+C_{0}}{C_{n}+C_{n-1}+\cdots+C_{0}} \\
& =\epsilon\left(1-\frac{C_{n}+C_{n-1}+\cdots+C_{n-m}}{C_{n}+C_{n-1}+\cdots+C_{0}}\right), m>m_{\mathrm{e}}=\text { constant },
\end{aligned}
$$

and hence $\lim _{n=\infty} Y(n)=0$. Thus the lemma becomes established.

* This is also true when each of the quantities $C_{0}, C_{1}, \cdots, C_{n}$ is a function of $n$, other conditions remaining the same, and the proof which follows will be found to apply equally to such cases. 
In particular, let us now take

$$
C_{n}=\frac{r(r+1) \cdots(r+n-1)}{n !}, r=\text { integer } \geqq 1,
$$

and place $c_{n}=s_{n}$, where $s_{n}$ represents the sum of the first $n+1$ terms of $(1)^{n}$. Then condition (4) is satisfied and (5) is at once seen to give the sum formula (2) of Cesàro. More generally, if $V_{n}$ be any function of $n$ such that the difference $V_{n}-V_{n-1}$ never decreases as $n$ increases ( $n$ sufficiently large), while at the same time

$$
\lim _{n=\infty} \frac{V_{n}-V_{n-1}}{V_{n}}=0,
$$

we may obtain in like manner a sum formula for the series (1) by taking $C_{n}=V_{n}-V_{n-1}$. In fact, we shall then have

$$
\frac{C_{n}+C_{n-1}+\cdots+C_{n-m}}{C_{n}+C_{n-1}+\cdots+C_{0}} \equiv(m+1) \frac{V_{n}-V_{n-1}}{V_{n}}
$$

so that condition (4) becomes satisfied, with which (5) yields the formula mentioned. In the special case leading to formula (2) it will be observed that

(7) $V_{n}=\frac{r(r+1) \cdots(r+n)}{n !} ; V_{n}-V_{n-1}=\frac{r(r+1) \cdots(r+n-1)}{n !}$,

so that

$$
\frac{V_{n}-V_{n-1}}{V_{n}}=\frac{r}{r+n} .
$$

We turn our attention therefore to the function $V_{n}$ defined by a general equation of the type

$$
\frac{V_{n}-V_{n-1}}{V_{n}} A_{n} ; \lim _{n=\infty} A_{n}=0 .
$$

A solution of this equation which reduces to (7) when

(case of Cesàro) is

$$
A_{n}=\frac{r}{r+n}
$$

$$
V_{n}=\coprod_{n=1}^{n}\left(\frac{1}{1-A_{n}}\right)
$$


Let us now consider the function $V_{n}$ obtained by taking

$$
A_{1}=\frac{1}{r+1}, \quad A_{n}=\frac{r}{r+\log n} \quad(n \geqq 2) .
$$

This function will necessarily satisfy (6) because of $(8)$ and will be such that $V_{n}-V_{n-1}$ is an ever increasing function of $n(n$ sufficiently large) since the result of increasing $n$ by 1 is to. multiply $V_{n}-V_{n-1}$ by

$$
\frac{A_{n+1}}{A_{n}} \cdot \frac{V_{n+1}}{V_{n}}=\frac{r+\log n}{\log (n+1)}>\frac{\log (n+r)}{\log (n+1)} \geqq 1 .
$$

Thus we reach the sum formulas

$$
s=\lim _{n=\infty} \frac{f_{p}(n, r) s_{0}+f_{p}(n-1, r) s_{1}+\cdots+f_{p}(n, r) s_{n}}{f_{p}(n, r)+f_{p}(n-1, r)+\cdots+f_{p}(0, r)}
$$

where

$$
f_{n}(n, r)=V_{n}-V_{n-1}=\frac{r(r+1)}{r+\log _{p} n} \prod_{n=2}^{n} \frac{r+\log _{p} n}{\log _{p} n} \quad(p=0,1)
$$

with $\log _{0} n=n$ (case of Cesàro) and $\log _{1} n=\log n$.

More generally, by taking

$$
A_{1}=\frac{r}{r+1}, \quad A_{n}=\frac{r}{r+\log _{p} n},
$$

where $\log _{0} n=n$ and $\log _{p} n=\log \log \cdots \log n$ ( $p$ times) we are led to a function $f_{p}(n, r)$ defined by the formula

$$
f_{p}(n, r)=\frac{r(r+1)}{r+\log _{p} n} \prod_{n=2}^{n} \frac{r+\log _{p} n}{\log _{p} n} \quad(p=0,1,2,3, \ldots)
$$

which function satisfies conditions $(a)$ and $(b)$ of $\S 2$.

In order to complete the proof of the theorem it remains. therefore but to show that the function $f_{p}(n, r)$ defined by (11). satisfies $(c)$ and $(d)$.

Recalling that

$$
\sum_{m=0}^{n} f_{p}(m, r)=\prod_{n=1}^{n}\left(\frac{1}{1-A_{n}}\right)=(r+1) \prod_{n=2}^{n} \frac{r+\log _{p} n}{\log _{p} n}
$$


we have

$$
\begin{aligned}
& \frac{\sum_{m=0}^{n} f_{p}(m, r)}{\sum_{m=0}^{n} f_{p}(m, r+1)}=\frac{r+1}{r+2} \prod_{n=2}^{n} \frac{r+\log _{p} n}{r+1+\log _{p} n} \\
& \quad=\frac{r+1}{r+2} \prod_{n=2}^{n}\left(1-\frac{1}{r+1+\log _{p} n}\right)
\end{aligned}
$$

and since the series

$$
\sum_{n=2}^{\infty} \frac{1}{r+1+\log _{p} n}
$$

is divergent, it follows from the well-known tests for convergence and divergence of infinite products that the last member of (12) approaches the limit 0 when $n=\infty$, with which condition (c) becomes satisfied.

Again we have

$$
\frac{\sum_{m=0}^{n} f_{p}(m, r)}{\sum_{m=0}^{n} f_{p+1}(m, 1)}=\prod_{n=2}^{n} \frac{\left(r+\log _{p} n\right) \log _{p+1} n}{\left(1+\log _{p+1} n\right) \log _{p} n}=\prod_{n=2}^{n}\left(1-g_{n}\right),
$$

where

$$
g_{n}=\frac{\frac{1}{1+\log _{p+1} n}-\frac{r}{r+\log _{p} n}}{\cdot 1-\frac{r}{r+\log _{p} n}} .
$$

For all values of $n$ we thus have

$$
g_{n}>\frac{\log _{p} n\left(1-\frac{r \log _{p+1} n}{\log _{p} n}\right)}{\left(r+\log _{p} n\right)\left(1+\log _{p+1} n\right)}>\frac{c}{\log _{p+1} n} ; c=\text { constant }
$$

and hence the series

$$
\sum_{n=2}^{\infty} g_{n}
$$

is divergent, with which condition $(d)$ becomes verified, thus completing the proof of the theorem. 